\title{
Preemptive analgesia in the "non-tube no fasting" fast track program for resectable esophageal carcinoma
}

\author{
Xianben Liu", Wentao Hao", Kun Gao", Wenqun Xing, Zongfei Wang, Haibo Sun, Yan Zheng \\ Department of Thoracic Surgery, The Affiliated Cancer Hospital of Zhengzhou University, Henan Cancer Hospital, Zhengzhou, China \\ Contributions: (I) Conception and design: X Liu, W Hao, Y Zheng; (II) Administrative support: X Liu, W Xing; (III) Provision of study materials \\ or patients: X Liu, Z Wang, H Sun; (IV) Collection and assembly of data: W Hao, K Gao; (V) Data analysis and interpretation: W Hao, K Gao, \\ Y Zheng; (VI) Manuscript writing: All authors; (VII) Final approval of manuscript: All authors. \\ \#These authors contributed equally to this work and should be considered as co-first authors. \\ Correspondence to: Yan Zheng, MD, PhD. Department of Thoracic Surgery, The Affiliated Cancer Hospital of Zhengzhou University, Henan Cancer \\ Hospital, Zhengzhou 450008, China. Email: sunnyzheng1@126.com.
}

Background: Surgery is the main treatment for esophageal cancer, but postoperative incision pain seriously reduces patients' quality of life. The aim of this study was to investigate the feasibility and effectiveness of preemptive analgesia of the upper abdomen with ropivacaine in the "non-tube no fasting" fast track recovery program for esophageal carcinoma.

Methods: We retrospectively analyzed the medical records of patients who underwent minimally invasive esophagectomy (MIE) from February 2014 to August 2014. Patients in the study group underwent a conventional analgesia program together with local infiltration injection of ropivacaine in the upper abdominal incision $30 \mathrm{~min}$ before the operation, while patients in the control group underwent the conventional analgesia program alone. Ropivacaine was injected locally around the planned surgical incision, including intradermally, subcutaneously, in the fascial muscles, and in the parietal peritoneum layers. Li's anastomosis method was performed in the neck after MIE. No indwelling chest tube or abdominal cavity tube was placed in any patients. The use of analgesic pumps in the two groups of patients was recorded.

Results: A total of 102 patients were enrolled in the study, with 52 patients in the study group and 50 patients in the control group. Patients in both groups completed the surgery successfully as planned, and the anesthesia methods and drugs used during the operation were the same. The surgical duration, blood loss, and the number of resected lymph nodes did not differ significantly between the two groups. Three patients in the study group and 10 patients in the control group used analgesia pumps $(\mathrm{P}=0.031)$. The visual analog scale (VAS) score at 30 days after surgery in the group with preemptive analgesia was significantly better than that in the control group $(\mathrm{P}=0.048)$.

Conclusions: Preemptive analgesia for the upper abdomen with ropivacaine in the "non-tube no fasting" fast track recovery program for esophageal carcinoma is feasible and worthy of promotion.

Keywords: Preemptive analgesia; minimally invasive esophagectomy (MIE); ropivacaine

Submitted Sep 19, 2021. Accepted for publication Dec 31, 2021.

doi: 10.21037/atm-21-4988

View this article at: https://dx.doi.org/10.21037/atm-21-4988

\section{Introduction}

China has the highest incidence of esophageal squamous cell carcinoma in the world. The 2020 global cancer burden data released by the International Agency for Research on Cancer of the World Health Organization showed that esophageal carcinoma ranks sixth in incidence [324,000] and fourth in mortality among malignant tumors in China, accounting for $7.1 \%$ and $10 \%$ of the total number of new cancers and cancer deaths, respectively. The global 5-year survival rate of esophageal carcinoma is $15-25 \%$. It is the 
sixth leading cause of death from cancer in men and a serious threat to human health that significantly reduces quality of life.

Surgery is still the main treatment mode for resectable esophageal carcinoma. Surgical treatment of esophageal carcinoma has a history of 100 years, and the first esophagectomy was successfully completed by Torek in 1913 (1). As medical science has advanced and our understanding of esophageal carcinoma has improved, esophageal carcinoma surgery has developed from the initial left thoracic approach to the right thoracic approach (2) and to the current minimally invasive esophagectomy (MIE) (3), and the surgical methods continue to improve. Open esophagectomy involves extensive trauma, severe pain, and high complication and mortality rates. MIE has the advantages of minimal trauma, minor pain, and a quick recovery, and the postoperative effect is equivalent to that of thoracotomy, and thus MIE has been rapidly promoted. Although tremendous efforts have been devoted to the alleviation of postoperative pain, despite significant success, some patients still cannot tolerate it. Therefore, other analgesic methods are urgently needed since no significant improvement has been achieved in the surgical method.

Pain is an important factor affecting the postoperative recovery of esophageal carcinoma patients. Our group has carried out multimodal analgesia research. As an important part of the "non-tube no fasting" fast track recovery program for esophageal carcinoma $(4,5)$, our team has tried preemptive analgesia by subcutaneous injection of ropivacaine in the upper abdomen half an hour before making the midline incision in the upper abdomen, the site with the highest postoperative pain score. As a result, the postoperative pain of the upper abdominal incision was significantly reduced. We present the following article in accordance with the STROBE reporting checklist (available at https://atm.amegroups.com/article/view/10.21037/atm$21-4988 / \mathrm{rc})$.

\section{Methods}

\section{General information}

In this retrospective cohort study, we reviewed MIE surgeries in the first ward of the Department of Thoracic Surgery of our hospital between February 2014 and August 2014. Some patients received neoadjuvant treatment before MIE, and some patients underwent surgical treatment directly. The inclusion criteria were as follows: (I) patients with esophageal squamous-cell carcinoma scheduled to undergo MIE and (II) patients subjected to the "nontube no fasting" fast track program. The exclusion criteria included the following: (I) patients who were converted to open thoracotomy or laparotomy during the surgery; (II) patients whose tumors were not resected, as shown by intraoperative examination; (III) patients who were transferred to the intensive care unit for more than $24 \mathrm{~h}$ after surgery; (IV) patients who used an analgesia pump immediately after the operation; (V) patients who were subjected to traumatic postoperative operations, such as thoracic catheter placement; and (VI) patients with early postoperative anastomotic leakage, pneumonia, or other serious complications. We compared patients in the study group and control group.

Patients in the study group received infiltration injection with one vial of ropivacaine $(75 \mathrm{mg}$ ) from the xiphoid process to the planned abdominal incision superior to the umbilicus $30 \mathrm{~min}$ before the operation. Patients in the control group did not receive any pretreatment.

The study was conducted in accordance with the Declaration of Helsinki (as revised in 2013). This study was approved by the Medical Ethics Committee of Henan Cancer Hospital (No. 2014xjs4), and individual consent for this retrospective analysis was waived.

\section{Surgical methods}

Li's manual anastomosis method was performed at the neck after MIE (5). The patient was placed in the left lateral decubitus position with a $30^{\circ}$ forerake and was subjected to thoracolaparoscopic esophagectomy and lymph node dissection. The thoracic esophagus was isolated, and the lymph nodes were resected. Then, the patient was switched to the supine position. A $2-3-\mathrm{cm}$ left cervical incision was made. The cervical esophagus was exposed and transected. A 4-cm-wide gastric conduit was made in the abdominal section with a linear cutting stapler (TLC, Ethicon, New Brunswick, NJ, USA). The gastric conduit was pulled up to the neck, and the gastric conduit and the distal esophagus were sutured according to Li's anastomosis method (5). The abdominal incision was generally approximately $5 \mathrm{~cm}$, and the size of the incision was adjusted appropriately according to the size of the patient.

\section{Intervention}

Patients in the study group were injected with one vial 
of ropivacaine $(75 \mathrm{mg})$ for infiltration analgesia from the xiphoid process to the planned abdominal incision superior to the umbilicus $30 \mathrm{~min}$ before the operation. Ropivacaine was injected locally around the planned surgical incision, including intradermally, subcutaneously, in the fascial muscles, and in the parietal peritoneum layers. The perioperative treatment of the two groups was the same, and no nasogastric or thoracic tubes were placed before or during surgery. The urethral catheter was removed, and the patient ate on the first day after surgery (4). Only the study group received preemptive analgesia, but the analgesic method used in both groups was fast-track recovery and conventional analgesia, including intercostal nerve freezing and routine intravenous administration of $100 \mathrm{mg}$ flurbiprofen axetil twice a day for 5-8 days after surgery.

\section{Observation indicators}

Postoperative analgesic pump use in each patient was recorded. The pain score of the median incision in the upper abdomen was evaluated 30 days after surgery. The analgesics in the analgesic pump were $0.2 \mathrm{~g}$ tramadol $+100 \mathrm{mg}$ flurbiprofen axetil.

\section{Pain assessment and analgesic pump application standards}

The degree of pain was assessed by a combination of the face (facial expression) pain rating scale and the visual analog scale (VAS). The severity of pain was expressed by numbers $0-10$, with 0 indicating no pain and 10 indicating the most intense pain imaginable. When the patient was in pain, the patients selected a number or facial expression that best represented their pain level, or the medical staff asked the patient, "How severe is your pain?", and then the medical staff selected a number according to the patient's description of the pain. The pain scores were grouped into mild [1-3], moderate [4-6], and severe pain [7-10]. An analgesia pump was used when the VAS score was $\geq 5$.

\section{Statistical analysis}

The measurement data are expressed as the mean \pm standard deviation (SD), and the count data are expressed as rates. A $t$-test was used to compare measurement data, and the $\chi^{2}$ test was used to compare count data. $\mathrm{P}<0.05$ was considered statistically significant. Statistical analyses were run by IBM SPSS Statistics 23.

\section{Results}

A total of 102 patients underwent MIE surgery in the first ward of the Department of Thoracic Surgery in our hospital between February 18, 2014, and August 29, 2014, and met the inclusion criteria, including 52 patients in the study group and 50 patients in the control group. Patients in the two groups did not differ significantly in sex, age, body weight, or surgical duration $(\mathrm{P}>0.05)$ (Table 1).

\section{Surgical situation}

All surgeries in both groups were successfully completed as planned. The anesthesia methods and drugs used during the operation were the same. The surgical duration, blood loss, and the number of lymph node dissections did not differ significantly between the two groups. No patient had an indwelling thoracic tube, but they each had an indwelling 19-gauge silicone mediastinal drainage tube (Table 2).

\section{Postoperative situation}

Three patients in the study group and 10 patients in the control group used an analgesia pump $(\mathrm{P}=0.031)$. The pain scores assessed at postoperative day 30 were significantly different between the two groups $(\mathrm{P}=0.048)$. The basic information of the study group and the control group did not significantly differ (Table 2).

\section{Discussion}

Pain can induce various negative effects in the body, such as effects on metabolism, endocrine activity, respiration, circulation, stress, nerves, and mental status, and has become an important medical problem affecting human health (6). In the era of thoracotomy and esophagectomy, before the advent of MIE, the incision length was generally 20-30 cm, muscles and even intercostal nerves were cut, rib fractures and even resection were possible during the surgery, and an indwelling thoracic tube was placed after esophagectomy. Many patients suffered intense pain after surgery, and many patients were discouraged when they heard they would need a thoracotomy. They were afraid of the surgery and chose other treatment methods, losing valuable time and their opportunity for the best treatment method.

Esophageal carcinoma is more common in elderly 
Table 1 Baseline demographic and clinical characteristics of esophageal carcinoma patients

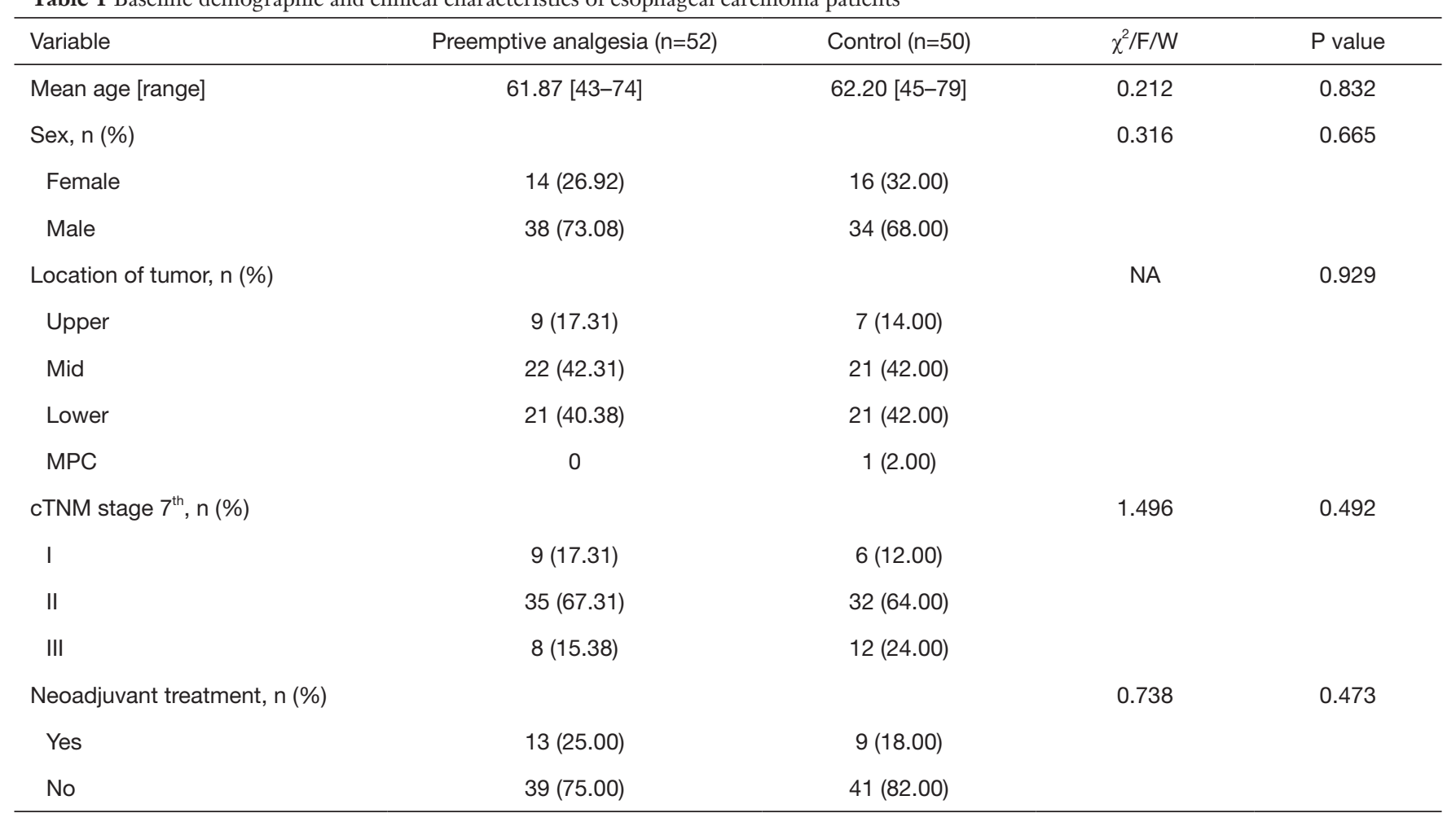

n, number; NA, not available; MPC, multiple primary cancer; cTNM, clinical tumor/node/metastasis stage.

patients, who often have chronic comorbidities such as chronic bronchitis and coronary heart disease. Postoperative pain can affect the deep breathing and postoperative coughing of patients, and secondary bronchial secretions can cause pneumonia and atelectasis, which may even lead to respiratory failure in severe cases. Patients are afraid of getting out of bed due to pain, which increases the risk of deep vein thrombosis and negatively impacts the recovery of bowel motility. MIE has markedly reduced but cannot completely prevent postoperative pain. Effective pain relief after thoracic and abdominal surgery has always been considered an important factor in ensuring a speedy recovery, reducing complications, and shortening hospitalization (6).

In our clinical observations before starting this study, we found that after MIE, the patient's pain mainly originated from the upper abdominal incision, while the pain in the chest and abdomen was almost negligible. Therefore, we thought that a preintervention for pain at the upper abdominal incision was particularly important.

The analgesic strategy of local infiltration can provide a strong analgesic effect and reduce the application of opioids after surgery, thereby reducing adverse events and shortening hospitalization (7-9). A prospective, doubleblinded, randomized, controlled trial in Australia reported by Huang et al. (10) showed that the local intraperitoneal injection of ropivacaine had an analgesic effect within $6 \mathrm{~h}$ after emergency laparoscopic appendectomy and reduced the dosage of postoperative opioid analgesics. In a prospective randomized control trial, Liang et al. (11) studied the efficacy and safety of different doses of ropivacaine for laparoscopy-assisted infiltration analgesia in patients undergoing laparoscopic cholecystectomy. The results showed that regardless of the dose used, local ropivacaine infiltration anesthesia at the incision successfully reduced the pain intensity of patients who underwent laparoscopic cholecystectomy. A study by Dam et al. (12) also found that preoperative block anesthesia of the bilateral quadratus lumborum muscles reduced postoperative opioid use and delayed the first opioid requirement after laparoscopic nephrectomy.

Ropivacaine is used in many surgeries because it is a long-acting anesthetic with a clear analgesic effect, a fast onset, few adverse effects, and an onset time after topical 
Table 2 Intraoperative and postoperative outcome

\begin{tabular}{|c|c|c|c|c|}
\hline Variable & Preemptive analgesia $(n=52)$ & Control $(n=50)$ & $\chi^{2} / F / W$ & $P$ value \\
\hline Mean operative time (SD, min) & $190.37(43.63)$ & $205.70(38.46)$ & 1.847 & 0.068 \\
\hline Mean blood loss (SD, mL) & $85.71(62.85)$ & $77.66(63.36)$ & 0.625 & 0.533 \\
\hline Median lymph nodes retrieved ( $\mathrm{n}$, range) & 20 [15-41] & 20 [15-48] & 0.713 & 0.478 \\
\hline \multicolumn{5}{|l|}{ Postoperative data } \\
\hline Median postoperative hospital stay (range, days) & 8 [5-45] & $10[6-31]$ & 0.300 & 0.765 \\
\hline Myocardial arrhythmia, n (\%) & $5(9.62)$ & $7(14.00)$ & & \\
\hline Pneumonia, n (\%) & $6(11.54)$ & $9(18.00)$ & & \\
\hline 30-day/in-hospital mortality, n (\%) & 0 & 0 & NA & NA \\
\hline 90-day mortality, n (\%) & 0 & 0 & NA & NA \\
\hline \multicolumn{5}{|l|}{ Pathological data } \\
\hline Type of carcinoma, n (\%) & & & NA & 0.523 \\
\hline Squamous cell carcinoma & 48 (92.31) & $44(88.00)$ & & \\
\hline Adenosquamous carcinoma & $2(3.85)$ & $1(2.00)$ & & \\
\hline Adenocarcinoma & $2(3.85)$ & $4(8.00)$ & & \\
\hline Small cell carcinoma & 0 & $1(2.00)$ & & \\
\hline III & $12(23.08)$ & $15(30.00)$ & & \\
\hline \multicolumn{5}{|l|}{ Pain control data } \\
\hline Analgesic pump, n (\%) & $3(5.77)$ & $10(20.00)$ & 4.642 & $0.031^{*}$ \\
\hline VAS 30 days after surgery, $\mathrm{n}(\%)$ & & & 3.911 & $0.048^{*}$ \\
\hline 0 & 44 (84.62) & $34(68.00)$ & & \\
\hline 1 & $8(15.38)$ & $16(32.00)$ & & \\
\hline
\end{tabular}

*, statistically significant $(\mathrm{P}<0.05)$. n, number; $\mathrm{SD}$, standard deviation; NA, not available; pTNM, tumor/node/metastasis; pCR, pathological complete response; VAS, visual analog scale.

application of 2-6 h (13). Our retrospective study also indicated that the 6-, 12-, 18-, and 24-h VAS scores of the study group, which received anesthetization of the upper abdominal incision by local ropivacaine infiltration, were significantly lower than those of the control group
$(\mathrm{P}<0.05)$. The use of analgesia pumps in the study group was lower than that of the control group. Although the postoperative time to get out of bed and postoperative bowel movement recovery time were similar between groups, local ropivacaine infiltration showed certain 
advantages. Our findings indicate that ropivacaine has a good topical analgesic effect and is beneficial to the patient's postoperative recovery, which is consistent with the aforementioned studies.

In the beginning of the 20th century, the concept of preemptive analgesia was proposed and was slowly applied in clinical medicine. Animal experiments have shown that preemptive analgesia can effectively prevent pain sensitization and reduce postoperative pain. Preemptive analgesia has a better effect than traditional postoperative pain relief methods. Preemptive analgesia can significantly reduce the application of opioid analgesics and analgesic pumps, which is consistent with our research findings (14). The greatest theoretical advantage of preemptive analgesia is that it effectively blocks chronic pain. The pain scores at 30 days after the operation in this study effectively confirm this theory and are consistent with reports from previous studies (14-16).

Although ropivacaine local infiltration anesthesia is effective for postoperative analgesia, the following issues still need to be addressed: (I) for patients undergoing neoadjuvant chemotherapy, further study is needed to investigate whether neoadjuvant chemotherapy has an impact on local anesthesia and analgesia. (II) How the concentration or dosage of ropivacaine impacts its analgesic effect is not clear. Liang et al. (11) showed that all different concentrations of ropivacaine had similarly good postoperative analgesic effects, suggesting that the analgesic effect of ropivacaine may not be closely related to its concentration. (III) The relationship between the dose and the analgesic effect needs to be further evaluated. In this study, we applied ropivacaine half an hour before the operation. Further study is needed to determine the optimal ropivacaine application time. (IV) Whether topical application of ropivacaine affects incision healing is not clear. We did not find any complications at the upper abdominal incision of the patients in the study group, suggesting that topical ropivacaine application may have no adverse effect on incision recovery. However, the sample size in this study was small, and further study is needed to confirm our findings.

\section{Acknowledgments}

This work was supported administratively by Professor Yin Li.

Funding: This work was supported by the National Natural Science Foundation of China, NSFC (grant number
82002521) and Natural Science Foundation of Henan Province (grant number 202300410389).

\section{Footnote}

Reporting Checklist: The authors have completed the STROBE Checklist. Available at https://atm.amegroups. com/article/view/10.21037/atm-21-4988/rc

Data Sharing Statement: Available at https://atm.amegroups. com/article/view/10.21037/atm-21-4988/dss

Peer Review File: Available at https://atm.amegroups.com/ article/view/10.21037/atm-21-4988/prf

Conflicts of Interest: All authors have completed the ICMJE uniform disclosure form (available at https://atm. amegroups.com/article/view/10.21037/atm-21-4988/coif). The authors have no conflicts of interest to declare.

Etbical Statement: The authors are accountable for all aspects of the work in ensuring that questions related to the accuracy or integrity of any part of the work are appropriately investigated and resolved. The study was conducted in accordance with the Declaration of Helsinki (as revised in 2013). This study was approved by the Medical Ethics Committee of Henan Cancer Hospital (No. 2014xjs4), and individual consent for this retrospective analysis was waived.

Open Access Statement: This is an Open Access article distributed in accordance with the Creative Commons Attribution-NonCommercial-NoDerivs 4.0 International License (CC BY-NC-ND 4.0), which permits the noncommercial replication and distribution of the article with the strict proviso that no changes or edits are made and the original work is properly cited (including links to both the formal publication through the relevant DOI and the license). See: https://creativecommons.org/licenses/by-nc-nd/4.0/.

\section{References}

1. Karamanou M, Markatos K, Papaioannou TG, et al. Hallmarks in history of esophageal carcinoma. J BUON 2017;22:1088-91.

2. Zheng Y, Li Y, Liu X, et al. Right Compared With Left Thoracic Approach Esophagectomy for Patients With Middle Esophageal Squamous Cell Carcinoma. Front 
Oncol 2020;10:536842.

3. Zheng Y, Li Y, Liu X, et al. Minimally Invasive Versus Open McKeown for Patients with Esophageal Cancer: A Retrospective Study. Ann Surg Oncol 2021;28:6329-36.

4. Sun HB, Li Y, Liu XB, et al. Early Oral Feeding Following McKeown Minimally Invasive Esophagectomy: An Openlabel, Randomized, Controlled, Noninferiority Trial. Ann Surg 2018;267:435-42.

5. Zheng Y, Li Y, Wang Z, et al. A video demonstration of the Li's anastomosis-the key part of the "non-tube no fasting" fast track program for resectable esophageal carcinoma. J Thorac Dis 2015;7:1264-8.

6. Echeverria-Villalobos M, Stoicea N, Todeschini AB, et al. Enhanced Recovery After Surgery (ERAS): A Perspective Review of Postoperative Pain Management Under ERAS Pathways and Its Role on Opioid Crisis in the United States. Clin J Pain 2020;36:219-26.

7. Das NT, Deshpande C. Effects of Intraperitoneal Local Anaesthetics Bupivacaine and Ropivacaine versus Placebo on Postoperative Pain after Laparoscopic Cholecystectomy: A Randomised Double Blind Study. J Clin Diagn Res 2017;11:UC08-12.

8. Siriwardana RC, Kumarage SK, Gunathilake BM, et al. Local infiltration versus laparoscopic-guided transverse abdominis plane block in laparoscopic cholecystectomy: double-blinded randomized control trial. Surg Endosc 2019;33:179-83.

9. Mont MA, Beaver WB, Dysart SH, et al. Local Infiltration Analgesia With Liposomal Bupivacaine Improves Pain Scores and Reduces Opioid Use After Total Knee Arthroplasty: Results of a Randomized Controlled Trial. J
Arthroplasty 2018;33:90-6.

10. Huang YY, Suthananthan AE, Hunt V, et al. The role of intraperitoneal ropivacaine in laparoscopic appendicectomy: a prospective, double-blinded randomized control Australian study. ANZ J Surg 2019;89:101-5.

11. Liang M, Chen Y, Zhu W, et al. Efficacy and safety of different doses of ropivacaine for laparoscopy-assisted infiltration analgesia in patients undergoing laparoscopic cholecystectomy: A prospective randomized control trial. Medicine (Baltimore) 2020;99:e22540.

12. Dam M, Hansen C, Poulsen TD, et al. Transmuscular quadratus lumborum block reduces opioid consumption and prolongs time to first opioid demand after laparoscopic nephrectomy. Reg Anesth Pain Med 2021;46:18-24.

13. Kwack JY, Ahn KH, Kwon YS. Postoperative pain control with ropivacaine following laparoscopic myomectomy: A randomized double-blind, pilot study. J Obstet Gynaecol Res 2019;45:871-6.

14. Kim MP, Godoy C, Nguyen DT, et al. Preemptive pain-management program is associated with reduction of opioid prescriptions after benign minimally invasive foregut surgery. J Thorac Cardiovasc Surg 2020;159:734-744.e4.

15. Pak DJ, Yong RJ, Kaye AD, et al. Chronification of Pain: Mechanisms, Current Understanding, and Clinical Implications. Curr Pain Headache Rep 2018;22:9.

16. Wang Y, Guo X, Guo Z, et al. Preemptive analgesia with a single low dose of intrathecal morphine in multilevel posterior lumbar interbody fusion surgery: a double-blind, randomized, controlled trial. Spine J 2020;20:989-97.
Cite this article as: Liu X, Hao W, Gao K, Xing W, Wang Z, Sun H, Zheng Y. Preemptive analgesia in the "non-tube no fasting" fast track program for resectable esophageal carcinoma. Ann Transl Med 2022;10(7):393. doi: 10.21037/atm-21-4988 